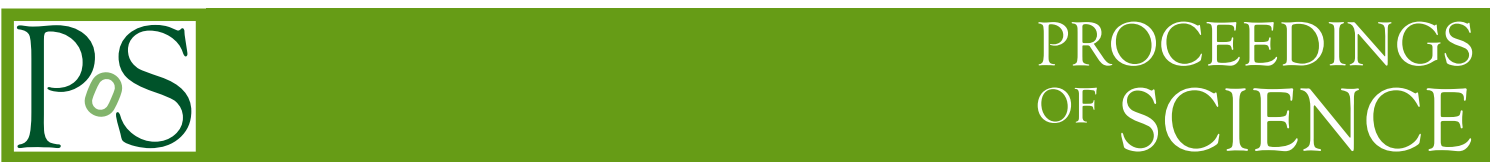

\title{
Searches for heavy resonances at ATLAS and CMS
}

\author{
Óscar González on behalf of the ATLAS and CMS Collaborations ${ }^{a, *}$ \\ ${ }^{a}$ CIEMAT, Avda. Complutense 40, E-28040 Madrid (Spain) \\ E-mail: oscar.gonzalez@ciemat.es
}

\begin{abstract}
We report on the most recent searches for new high-mass resonances decaying into leptons, quarks or gluons performed by the ATLAS and CMS Collaborations using the full data set collected during the Run 2 of the LHC. Although no significant discrepancy is observed with respect to the Standard Model expectation, the results provide the strongest constraints for the presence of new physics in several extensions of the Standard Model, for both model-dependent and model-independent resonance production.
\end{abstract}

The Eighth Annual Conference on Large Hadron Collider Physics-LHCP2020

25-30 May, 2020

online

${ }^{*}$ Speaker 


\section{Introduction and experimental context}

Many proposed extensions of the Standard Model (SM), motivated to account for the shortcomings of such a model, introduce new particles and interactions with reference scales above the so-called electroweak scale, where the SM is giving a good description of the observation in the data. Those scales may be within the reach of the energy regime of the LHC, where the two largest collaborations, ATLAS and CMS, are able to use their available data from the Run 2, around $140 \mathrm{fb}^{-1}$ of integrated luminosity, to search for the possible presence of new particles or their effects even if their production cross sections are small.

These two experiments are investigating the detection of new possible massive particles by means of their decay products, which are assumed to be particles belonging to the SM that are identified by the ATLAS and CMS detectors $[1,2]$. Thanks to their excellent performance during the Run 2 of the LHC, in terms of coverage, efficiency and resolution, these experiments are well suited to look for new particles at masses at the 1-10 $\mathrm{TeV}$ range.

\section{Description of the experimental analyses}

Being located at a hadron collider, ATLAS and CMS have good chances to discover new particles when they decay into electrons and muons. This kind of searches are advantageous due to the low backgrounds and the good resolution and efficiency to identify electrons and muons when they have a large transverse momentum. A search by CMS [3] is performed by selecting events with two oppositely-charged electrons or muons and reconstructing the invariant mass. For that selection the main background is given by Drell-Yan production that is easily estimated by using the SM predictions and the detector simulation. Normalizing the expectations to the $\mathrm{Z}$ boson resonant peak it is possible to reduce the systematic uncertainties.

In the analysis, CMS observed a slight excess for dielectron events having a mass larger than 1.8 $\mathrm{TeV}$, but it is not significant enough. The result is therefore used to set limits on the production cross section of a new particle, using the production cross section of the $\mathrm{Z}$ boson as reference. Such a limit is obtained for a model-independent signal and shown in Figure 1, where it is explicitly compared to the expectations for the sequential SM $Z^{\prime}$ and the $Z_{\phi}^{\prime}$ boson predicted by the $E_{6}$ model [4].

Along with the search with dileptons, both collaborations also looked for resonances in the invariant dijet mass spectrum as a signature for a new particle decaying into quarks and/or gluons. This suffers from higher backgrounds but it has the advantage that if the particle is produced in the proton-proton collisions of the LHC, it should have a decay into jets, and usually with high cross sections even at high masses. The sensitivity to possible leptophobic particles is the main advantage of the searches previously mentioned. Despite of the challenges, ATLAS has studied [5] the dijet mass spectrum in the range between 1.1 and $8 \mathrm{TeV}$ using events with two jets that are divided into several categories depending on their b-tagging content and the value of the halfrapidity separation between the two jets. The use of a sliding-window fitting technique applied to the data in the signal region allows to have an estimation of the SM background, dominated by the continuous production of dijet events from the strong interaction. No significant excess is found in the analysis and the data are used to constrain many signal models and even a generic hypothetical 
signal modelled as a Gaussian peak in the particle-level distribution of the dijet invariant mass. A large increase in sensitivity with respect to the previous result [6] is observed, coming mostly from the improvement in b-tagging which used a deep-learning neural network for the first time by the ATLAS collaboration.

CMS has performed an equivalent study for dijet invariant masses above $1.8 \mathrm{TeV}$ [7]. In this case, a dedicated technique to combine further jets with the two leading ones into so-called "wide jets" reduces the sensitivity of the search to gluon final-state radiation by providing a much improved resolution in the reconstructed mass. Additionally two independent techniques are used to estimate the SM backgrounds: a previously used method by fitting the entire dijet mass spectrum with a smooth function and a ratio method combining the information from control regions and a transfer factor estimated from simulation and constrained by the data in a second control region. The latter technique yields a more rigid background prediction with significantly smaller systematic uncertainties. A large increase in sensitivity is achieved this way especially for broad resonances with a width much larger than the experimental resolution.

Both methods are able to describe the observed distribution in the data, with no significant discrepancy that could be attributed to a new resonance. The results are interpreted in the context of several models predicting massive particles that decay into quarks or gluons and limits are obtained and classified in terms of the nature of the jets and other properties of the hypothetical particle, so they may be applied to a large variety of models.

In order to increase the sensitivity for resonances decaying to quarks and/or gluons, ATLAS has produced a study looking for heavy mass resonances produced in association with a high transversemomentum electron or muon [8]. This introduces some model dependence on the possible signal, but allows the search for resonances with lower masses, since the presence of the lepton allows the reduction of the background, specially in the online selection step. Figure 2 shows the dijet invariant mass distribution for the leading jets in this kind of events containing at least a lepton, usually produced via the presence of a weak boson or a top quark. The covered range in this case is from $220 \mathrm{GeV}$ to $6.3 \mathrm{TeV}$, with gained sensitivity in the lower edge with respect to the aforementioned result. Still a good agreement is observed between the data and the estimated background, and the results are interpreted to set constraints on generic signals, described via a Gaussian distribution with various widths. Furthermore, some limits are set in specific models with more complex topologies. The limits obtained here are complementary to the results from more dedicated analyses, more optimized to exploit the specific final state topology.

Another analysis by ATLAS in the context of dijet resonances is targeting new heavy particles decaying into a top-antitop pair [10], using the fully hadronic decay mode in boosted topologies, obtained for resonance masses larger than $1.4 \mathrm{TeV}$. In this regime the identification of the three-prong internal structure of the jets originating from the top-quark decay is exploited to gain sensitivity to a possible anomalous production of such boosted top-quark pairs. Additionally the use of b-tagged jets to identify the presence of the $b$ quarks from the top-quark decay also reduces the background and increases the sensitivity.

The background estimation, obtained with a smooth function fitting the SM expectation from simulated samples, adjusted by means of data-based corrections, describes the data well and there 


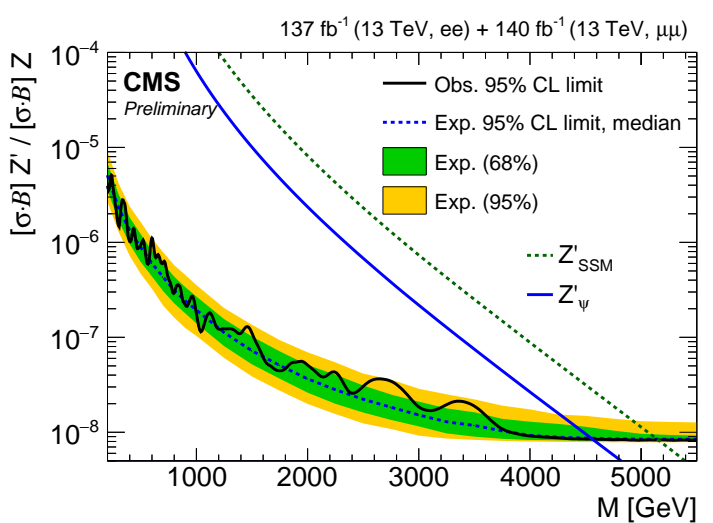

Figure 1: The upper limits at $95 \% \mathrm{CL}$ on the product of production cross section and branching fraction for a spin- 1 resonance with a width equal to $0.6 \%$ of the resonance mass, relative to the product of production cross section and branching fraction of the $\mathrm{Z}$ boson, for the combination of the dielectron and dimuon channels. The shaded bands correspond to the 68 and $95 \%$ quantiles for the expected limits. Theoretical predictions for two reference models are shown for comparison. Plot taken from [3]

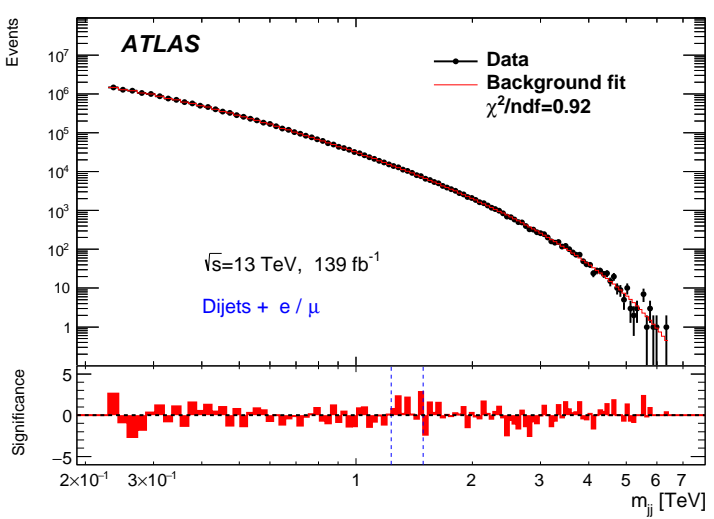

Figure 2: Dijet invariant-mass distribution from events in the ATLAS dataset with a high- $p_{T}$ lepton, calculated from the two leading jets. Also shown is the result of the fit with a five-parameter background function. The lower panel shows the bin-by-bin significances of deviations from the background hypothesis. The largest deviation reported by BumpHunter [9] is indicated by the vertical dashed lines. The global p-value of this deviation is 0.31 . Plot taken from [8]

is no significant excess, thus the results are used to set new constraints, using the topcolor-assistedtechnicolor model [11] as a benchmark for the results. Also in this case the sensitivity improvement with respect to the previous result [12] is well beyond that due to the luminosity increase.

\section{Conclusions}

In summary, several results from analyses searching for high-mass resonances performed by ATLAS and CMS using the full Run-2 dataset have been presented. No sign of new particles or interactions has been observed in the several final states under study, with sensitivities to masses that range from above the electroweak scale up to several $\mathrm{TeV}$. The results have been interpreted to set limits in specific models and in several model-independent frameworks. Additionally the analysis sensitivity goes beyond that expected from just the increase of luminosity due the analysis improvements. This motivates good expectations for future searches with the new datasets and improved analysis techniques during the Run 3 and the high-luminosity stages of the LHC program.

\section{References}

[1] ATLAS Collaboration, JINST 3 (2008) S08003. http://dx.doi.org/10.1088/1748-0221/3/08/S08003.

[2] CMS Collaboration, JINST 3 (2008) S08004. http://dx.doi.org/10.1088/1748-0221/3/08/S08004. 
[3] CMS Collaboration, Report CMS-PAS-EXO-19-019 (2019). http://cds.cern.ch/record/2684757

[4] A. Leike, Phys. Rept. 317 (1999) 143. https://doi.org/10.1016/S0370-1573(98)00133-1; J. L. Hewett and T. G. Rizzo, Phys. Rept. 183 (1989) 193. https://doi.org/10.1016/03701573(89)90071-9.

[5] ATLAS Collaboration, JHEP03 (2020) 145. https://doi.org/10.1007/JHEP03(2020)145.

[6] ATLAS Collaboration, Phys. Rev. D98 (2018) 032016. https://doi.org/10.1103/PhysRevD.98.032016.

[7] CMS Collaboration, JHEP05 (2020) 033. https://doi.org/10.1007/JHEP05(2020)033.

[8] ATLAS Collaboration, JHEP06 (2020) 151. https://doi.org/10.1007/JHEP06(2020)151.

[9] G. Choudalakis, in Proceedings, PHYSTAT 2011 Workshop on Statistical Issues Related to Discovery Claims in Search Experiments and Unfolding (2011), https://arxiv.org/abs/1101.0390.

[10] ATLAS Collaboration, Report CERN-EP-2020-055. https://arxiv.org/abs/2005.05138.

[11] C.T. Hill and S.J. Parke, Phys. Rev. D49 (1994) 4454. https://arxiv.org/abs/hep-ph/9312324; C.T. Hill, Phys. Lett. B345 (1995) 483. https://arxiv.org/abs/hep-ph/9411426; R.M. Harris and S. Jain, Eur. Phys. J. C72 (2012) 2072. https://arxiv.org/abs/1112.4928.

[12] ATLAS Collaboration, Phys. Rev. D99 (2019) 092004. https://doi.org/10.1103/PhysRevD.99.092004. 